\title{
Ameliorating effects of gypenosides on chronic stress-induced anxiety disorders in mice
}

\author{
Ting Ting Zhao ${ }^{1}$, Keon Sung Shin ${ }^{1}$, Hyun Sook Choi ${ }^{2}$ and Myung Koo Lee ${ }^{1 *}$
}

\begin{abstract}
Background: Ethanol extract from Gynostemma pentaphyllum (GP) shows anti-stress and anxiolytic functions in mice, and also protects dopamine neurons in 6-hydroxydopamine-lesioned rat model of Parkinson's disease. In addition, gypenosides (the gypenoside-enriched components of GP, GPS) have a protective effect on 1-methyl-4-phenyl1,2,3,6-tetrahydropyridine-induced mouse model of Parkinson's disease. In this study, the ameliorating effects of GPS on chronic stress-induced anxiety disorders in mice were investigated.
\end{abstract}

Methods: Mice were orally treated with GPS (100 and $200 \mathrm{mg} / \mathrm{kg}$ ) once a day for 10 days, followed by exposure to electric footshock (EF) stress ( $0.6 \mathrm{~mA}, 1 \mathrm{~s}$ every $5 \mathrm{~s}, 3 \mathrm{~min}$ ). After the final administration of either GPS, water extract of GP (GP-WX) or ethanol extract of GP (GP-EX, positive control), the behavioral tests such as elevated plus-maze, marble burying and locomotor activity tests, and the biochemical parameters including dopamine, serotonin and corticosterone levels, and c-Fos expression were examined.

Results: Treatment with GPS (100 and $200 \mathrm{mg} / \mathrm{kg}$ ) increased the number of open arm entries and the time spent on open arms in elevated plus-maze which were reduced by chronic EF stress. GPS (100 and $200 \mathrm{mg} / \mathrm{kg}$ ) reduced the number of marbles buried which increased by chronic EF stress. In these states, the brain levels of dopamine and serotonin decreased by chronic EF stress and they were recovered by GPS. The serum levels of corticosterone increased by chronic EF stress were also reduced by GPS (100 and $200 \mathrm{mg} / \mathrm{kg}$ ). Finally, chronic EF stress-induced c-Fos expression was markedly reduced by GPS (100 and $200 \mathrm{mg} / \mathrm{kg}$ ) in the brain. GPS (100 and $200 \mathrm{mg} / \mathrm{kg}$ ) also showed an equivalent efficacy on anxiolytic functions, as compared with GP-EX (50 mg/kg). However, GP-WX (50 mg/kg) showed a less effect on anxiety disorders than GP-EX (50 mg/kg) and GPS (100 and $200 \mathrm{mg} / \mathrm{kg}$ ).

Conclusion: These results suggest that GPS (100 and $200 \mathrm{mg} / \mathrm{kg}$ ) has anxiolytic effects on chronic EF stress-induced anxiety disorders by modulating dopamine and serotonin neuronal activities, c-Fos expression and corticosterone levels. GPS may serve as a phytonutrient in chronic stress-induced anxiety disorders.

Keywords: Gypenosides, Chronic stress-induced anxiety disorders, Elevated plus-maze, Dopamine, Serotonin, Corticosterone

\section{Background}

Slight or acute stress and repeated long-lasting or chronic stress are characterized by the physiological changes that occur in response to novel or threatening stimuli. Chronic stress has been linked to the pathophysiology of various psychiatric disorders, including anxiety disorders and depression [1]. In the conditions of chronic stress-induced anxiety disorders and depression, the levels of dopamine

\footnotetext{
* Correspondence: myklee@chungbuk.ac.kr

'College of Pharmacy and Research Center for Bioresource and Health, Chungbuk National University, Cheongju, Chungbuk 28644, Republic of Korea

Full list of author information is available at the end of the article
}

and serotonin in the brain are decreased. In contrast, the serum levels of glucocorticoids such as corticosterone and cortisol are increased by various chronic stresses, and this is mediated by the hypothalamic-pituitary-adrenal axis [2]. In addition, c-Fos protein, which is an immediate early gene, is stimulated against chronic stress in various brain regions of mice and rats, especially paraventricular nuclei (PVN) of hypothalamus $[3,4]$.

A number of chronic stress models, including electric footshock (EF) stress, forced swimming, noise stimuli and immobilization have been employed to induce anxiety disorders $[5,6]$. In the field of anxiety disorders induced 
by chronic stress, the elevated plus-maze and marble burying tests are widely used in response to threatening stimuli $[7,8]$. Spontaneous locomotor activity can be also decreased by chronic stress [9].

The traditional medicinal herb, Gynostemma pentaphyllum Makino (GP, Cucurbitaceae) has been identified with about 90 dammarane-type glycoside derivatives (called Gynostemma total saponins, gypenosides or gynosaponins) and flavonoids [10]. Previously, ethanol extract from GP (GP-EX) has been reported to have anti-stress and anxiolytic functions in mice [6]. GP-EX also protects dopamine neurons in 6-hydroxydopamine-lesioned rat model of Parkinson's disease (PD) [11]. In addition, the gypenosideenriched components (gypenosides, GPS) have a protective effect on 1-methyl-4-phenyl-1,2,3,6-tetrahydropyridine-induced mouse model of PD [12, 13].

In this study, in order to further define the anxiolytic function of GP, the pharmacological effects of GPS, which was obtained from GP $[12,13]$, on chronic electric footshock (EF) stress-induced anxiety disorders in mice were investigated. In addition, the anxiolytic functions by GPS were compared with those by water extract of GP (GPWX) and GP-EX (positive control).

\section{Methods}

\section{Chemicals}

Dopamine, norepinephrine, serotonin and 5-hydro xyindoleacetic acid (HIAA) were purchased from Sigma (St Louis, MO, USA). Corticosterone kit was obtained from USCN Life Sci. (E0504 m, Wuhan, China) and c-Fos antibody was from Santa Cruz Biotechnology (Santa Cruz, CA, USA). All other chemicals were of analytical grade.

\section{Preparation of GPS and GP extract}

GPS were purchased from Ankang Dongke Maidisen Co. (purity $>98 \%$, determined by UV) (Xi'an, China) [12, 13]. GP was obtained from Wonkwang Food Manufacturing Co. (Geochang, Korea) and a voucher specimen of the herbal leaves of GP was deposited at the herbarium of the College of Pharmacy, Chungbuk National University (Cheongju, Korea). The air-dried leaves of GP (5 kg) were extracted twice with distilled water at $80{ }^{\circ} \mathrm{C}$ for $3 \mathrm{~h}$ (GPWX) and $80 \%$ ethanol at $25^{\circ} \mathrm{C}$ for $12 \mathrm{~h}$ (GP-EX), respectively, and then the each extract was evaporated to dryness $[6,10,14]$. Ultimately, $390 \mathrm{~g}(7.8 \%$, w/w) of GP-WX and $490 \mathrm{~g}(9.8 \%, \mathrm{w} / \mathrm{w})$ of GP-EX, which were determined using high performance liquid chromatography (HPLC), were obtained.

\section{Experimental design}

Mice (ICR, male, 20-25 g) were obtained from Samtako Co. (Osan, Korea). Mice were housed two per cage in a temperature and humidity controlled environment (12-h light/dark cycle, $23 \pm 2{ }^{\circ} \mathrm{C}, 60 \pm 2 \%$ ), with ad libitum access to standard mouse food and water. This study was approved by the Animal Ethics Committee of Chungbuk National University (Approval No., CBNU-481-12-01).

The experiment was performed by 2 separate subsets for the elevated plus-maze and marble burying tests including the biochemical analyses, and for the spontaneous locomotor activity test including the immunohistochemical analyses. Each subset contained 16 groups (8-10 animals per group): half groups were exposed to the chronic EF stress and half were not. Each eight groups were orally treated for 10 days before $4 \mathrm{~h}$ the exposure of EF stress as follow: control group was the group that received saline $(0.9 \%)$, each GPS-treated group was the group that received one of the different doses of GPS $(30,50,100,200$, or $400 \mathrm{mg} / \mathrm{kg}$ ), GP-WX treated group was the group that received GP-WX (50 mg/kg), and GP-EX-treated group was the group that received GP-EX (50 mg/ $/ \mathrm{kg})$. Stress was the group which was exposed to chronic EF stimuli with either saline (0.9\%), GPS, GP-WX or GP-EX for 10 days. GP-EX was used as a positive control [6].

In the first experiments, the elevated plus-maze and marble burying tests were performed on the final day (day-10) at 30 min after the exposure of EF stress. The elevated plus maze test was carried out before the marble burying test to approximate a stressor (the interval time, 15-20 $\mathrm{min}$ ) [15]. After the behavioral tests, the mice were sacrificed to obtain brain tissues and serum for biochemical analyses. In the second experiments, the spontaneous locomotor activity test was carried out every day after $30 \mathrm{~min}$ of the exposure of chronic EF stress and then, the mice were also sacrificed to obtain brain tissues for immunehistochemical analyses.

\section{The exposure of chronic EF stress}

The mice were placed individually in the electrified shock chamber for the exposure to chronic stress and they received unavoidable EF stimuli (intensity, $0.6 \mathrm{~mA}, 1 \mathrm{~s}$ every $5 \mathrm{~s}$, periods, $3 \mathrm{~min}$ ) at 14:00 every day for 10 days using an electric shock generator (Seil Electric Co., Taejeon, Korea) [16].

\section{The elevated plus-maze test}

The elevated plus-maze apparatus consists of four arms: two open arms $(30 \times 5 \mathrm{~cm})$ and two closed arms of the same size, with 16-cm-high black walls elevated $45 \mathrm{~cm}$ above the floor. The open and closed arms were connected via a central square $(5 \times 5 \mathrm{~cm})$ to form a plus sign. The 5-min pre-adaptation period of 1 time for the last 3-days was conducted before the main test [17]. The number of open arm entries and the time spent on the open arms were recorded during a 5-min test period after the adaptation period of 5 -min by a video camera 
connected to a SMART video-tracking system (Panlab S.I., Barcelona, Spain) [7].

\section{The marble burying test}

The marble burying test was placed in a cage measuring $33 \mathrm{~cm} \times 21 \mathrm{~cm} \times 19 \mathrm{~cm}(1 \times \mathrm{w} \times \mathrm{h})$, containing bedding that was $5 \mathrm{~cm}$ in depth, with twenty marbles in a $4 \times 5$ arrangement along the same short wall of the cage. The 15-min pre-adaptation period for the last 3-days was applied before the main test [18]. Testing was conducted for a 30-min period under red light and white noise. After a $30 \mathrm{~min}$, the number of marbles buried was recorded and marbles were considered to be buried if at least two thirds of their surface was covered with bedding [8].

\section{The spontaneous locomotor activity test}

The locomotor activity was determined by a tilting-type ambulometer (Model AMB-10, O'Hara, Tokyo, Japan). Each mouse was placed in the round activity cage $(20 \mathrm{~cm}$ in diameter and $18 \mathrm{~cm}$ in depth) and after an adaptation period of $10 \mathrm{~min}$, the counts of horizontal movements of mice inside the cage were automatically recorded in $30 \mathrm{~min}$ [6].

\section{Measurement of dopamine, serotonin and corticosterone levels}

The whole brains were dissected out and frozen in a $-70{ }^{\circ} \mathrm{C}$ until analysis. For the measurement of dopamine levels, the brain tissues were homogenized in perchloric acid $(300 \mu \mathrm{L}$, $1 \mathrm{M}$ ) and isoproterenol (100 pmol, internal standard), and dopamine levels were measured by an HPLC system [19]. For the measurement of serotonin levels, the brain tissues were also homogenized in trichloroacetic acid $(500 \mu \mathrm{L}, 0.3 \mathrm{M})$ and HIAA (300 pmol, internal standard), and serotonin levels were measured by an HPLC system [6].

Blood was collected from the heart of sacrificed mice, and was then centrifuged to obtain a serum. Corticosterone levels were assessed using an enzyme-linked immunosorbent assay kit [6].

\section{Immunohistochemistry of c-Fos}

Mice were transcardially perfused with a paraformaldehyde solution ( $4 \%$ in $0.1 \mathrm{M}$ phosphate buffered saline, $\mathrm{pH}$ 7.4), and then the brains were removed. Coronal sections $(35 \mu \mathrm{m})$ at the PVN regions were processed for c-Fos immunocytochemistry using a polyclonal rabbit anti-c-Fos antibody (1:500) and a biotinylated goat antirabbit antibody (1:1,000; Vector Laboratories, Burlingame, $\mathrm{CA})$. The sections were incubated in avidin:biotinylated enzyme complex kit and exposed to 3,3'-diaminobenzidine kit for detection. c-Fos-positive cells in the PVN regions were counted using an image analysis system (Axiovision software, Carl Zeiss MicroImaging, GmbH, Jena, Germany) using a microscope (100X magnification) (Zeiss Axiophot, Carl Zeiss MicroImaging).

\section{Statistical analysis}

Data were analyzed with one-way analysis of variance (ANOVA) followed by Tukey's test for evaluating the dosedependent effects, two-way ANOVA followed by Tukey's test for evaluating the effects on chronic stress and threeway ANOVA by Tukey's test for evaluating the effect on between the three factors, doses, un-stressed and stressed stimuli, and days. All data represented as means \pm S.E.M. with $p$ values $<0.05$ being considered statistically significant.

\section{Results}

\section{Effects of GPS on elevated plus-maze}

In the un-stressed groups, treatment with GPS (30, 50, 100, 200 and $400 \mathrm{mg} / \mathrm{kg}$ ) once a day for 10 days did not alter both the number of open arm entries and the time spent on open arms in the elevated plus-maze test, compared with control group (Fig. 1). In contrast, the number of open arm entries and the time spent on open arms showed the significant differences between the un-stressed groups and stressed groups ((A) $\mathrm{F}=9.411$, (B) $\mathrm{F}=16.352 ; p<0.05$ ) (Fig. 1). The number of open arm entries and the time spent on open arms also decreased by $31.5 \%(p<0.01)$ and $48.4 \%(p<0.01)$ by chronic EF stress, compared with control group (Fig. 1a and b). However, the number of open arm entries increased by $19.6 \%(p<0.05)$ and $18.6 \%$ $(p<0.05)$, respectively by treatment with GPS (100 and $200 \mathrm{mg} / \mathrm{kg}$ ) for 10 days, compared with chronic EF-stressed group (Fig. 1a). However, GPS (30, 50 and $400 \mathrm{mg} / \mathrm{kg}$ ) did not show significant effects $(7.4 \%, 12.0 \%$ and $12.7 \%$; $p>0.05$ ) (Fig. 1a). The times spent on open arms reduced by chronic EF stress also increased by $20.4 \%$ $(p<0.05)$ and $18.1 \%(p<0.05)$, respectively by treatment with GPS (100 and $200 \mathrm{mg} / \mathrm{kg}$ ), compared with chronic EF-stressed group (Fig. 1b). However, GPS (30, 50 and $400 \mathrm{mg} / \mathrm{kg}$ ) did not show significant effects (10.3\%, $14.2 \%$ and $16.5 \% ; p>0.05$ ) (Fig. 1b).

The number of open arm entries and the time spent on open arms, which decreased by chronic EF stress, were also recovered by $18.8 \%(p<0.05)$ and $21.1 \%(p<0.05)$, respectively by treatment with GP-WX $(50 \mathrm{mg} / \mathrm{kg})$ for 10 days, compared with chronic EF-stressed group (Fig. 1a and b).

\section{Effects of GPS on marble burying}

In the un-stressed groups, treatment with GPS (30$400 \mathrm{mg} / \mathrm{kg}, 10$ days) had no effect on the number of marbles buried. The number of marbles buried increased by $60.2 \%(p<0.01)$ by exposure to chronic EF stress, compared with control group (Fig. 2). However, the number of 

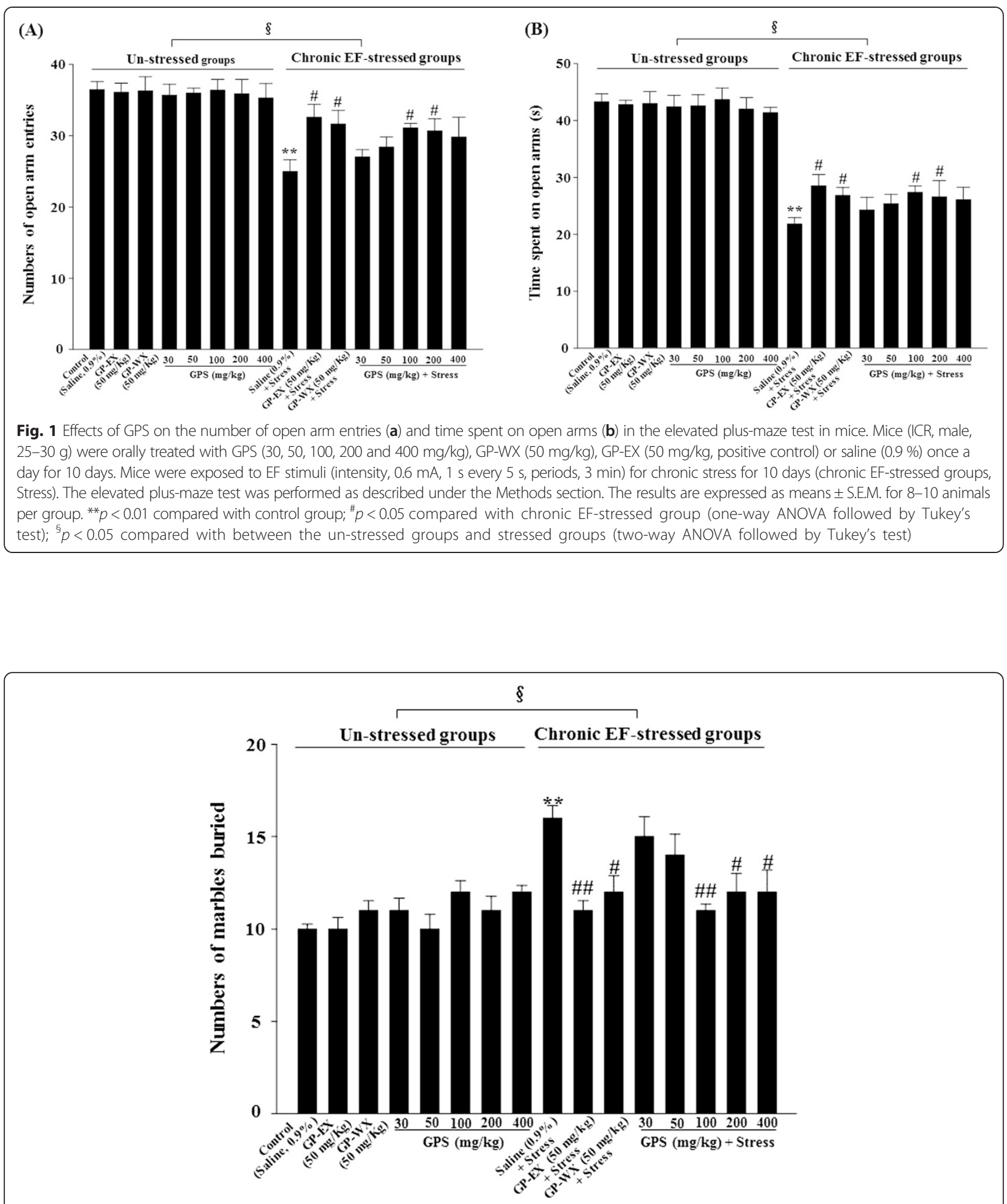

Fig. 2 Effects of GPS on the number of marbles buried in the marble burying test in mice. Mice (ICR, male, 25-30 g) were orally treated with GPS (30, 50, 100, 200 and 400 mg/kg), GP-WX (50 mg/kg), GP-EX (50 mg/kg, positive control) or saline (0.9 \%) once a day for 10 days. Mice were also exposed to EF stimuli (intensity, $0.6 \mathrm{~mA}, 1 \mathrm{~s}$ every $5 \mathrm{~s}$, periods, $3 \mathrm{~min}$ ) for chronic stress for 10 days (chronic EF-stressed groups, Stress). The marble burying test was performed as described under the Methods section. The results are expressed as means \pm S.E.M. for $8-10$ animals per group. ${ }^{* *} p<0.01$ compared with control group; ${ }^{\#} p<0.05,{ }^{\# \#} p<0.01$ compared with chronic EF-stressed group (one-way ANOVA followed by Tukey's test); ${ }^{\S} p<0.05$ compared with between the un-stressed groups and stressed groups (two-way ANOVA followed by Tukey's test) 
marbles buried of the stressed groups showed the significant differences between the un-stressed groups and stressed groups $(\mathrm{F}=5.284, p<0.05)$ (Fig. 2). The number of marbles buried also decreased by $31.3 \%(p<0.01)$, $25.2 \%(p<0.05)$ and $24.9 \%(p<0.05)$, respectively by treatment with GPS (100, 200 and $400 \mathrm{mg} / \mathrm{kg})$ for 10 days, compared with chronic EF-stressed group (Fig. 2). However, GPS (30 and $50 \mathrm{mg} / \mathrm{kg}$ ) did not show significant effects $(6.2 \%$ and $12.4 \% ; p>0.05)$ (Fig. 2).

In addition, the number of marbles buried decreased by $25.1 \%(p<0.05)$ by treatment with GP-WX $(50 \mathrm{mg} / \mathrm{kg})$, compared with chronic EF-stressed group (Fig. 2).

\section{Effects of GPS on spontaneous locomotor activity}

In the un-stressed groups, treatment with GPS (30$400 \mathrm{mg} / \mathrm{kg}, 10$ days) exhibited no significant effect on the spontaneous locomotor activity. The spontaneous locomotor activities showed the significant differences between the un-stressed groups and stressed groups (stress treated with saline and stress treated with GPS 100, $200,400 \mathrm{mg} / \mathrm{kg})(\mathrm{F}=2.469, p<0.05)$. GPS $(100 \mathrm{mg} / \mathrm{kg})$ was shown as a representative (Fig. 3). The spontaneous locomotor activities also showed a significant three-way interaction between factors, doses, un-stressed and stressed stimuli, and days (day-1 and day-10) $(\mathrm{F}=2.041$, $p<0.05$ ) (Fig. 3).

In the stressed groups, the spontaneous locomotor activities showed biphasic results. The spontaneous locomotor activities by exposure to acute EF stress for 2-4 days increased by $16.8 \%(p<0.05)$ (Fig. 3), compared with control group and baseline. However, after exposure to chronic EF stress for 10 days, spontaneous locomotor activity decreased by $18.1 \%(p<0.05)$, compared with control group, which was increased $15.4(p<0.05)$ and $15.8 \%$ $(p<0.05)$, respectively by GPS (100 and $200 \mathrm{mg} / \mathrm{kg})$, compared with chronic EF-stressed group (Fig. 3). However, GPS $(30,50$ and $400 \mathrm{mg} / \mathrm{kg}$ ) did not show significant effects $(8.7 \%, 9.4 \%$ and $12.8 \% ; p>0.05)$ (Fig. 3).

Treatment with GP-WX $(50 \mathrm{mg} / \mathrm{kg})$ also increased spontaneous locomotor activity by $15.0 \%(p>0.05)$, compared with chronic EF-stressed group (Fig. 3).

\section{Effects of GPS on the levels of dopamine and serotonin in} the brain

In the un-stressed groups, treatment with GPS (30$400 \mathrm{mg} / \mathrm{kg}$ ) for 10 days did not alter the levels of dopamine

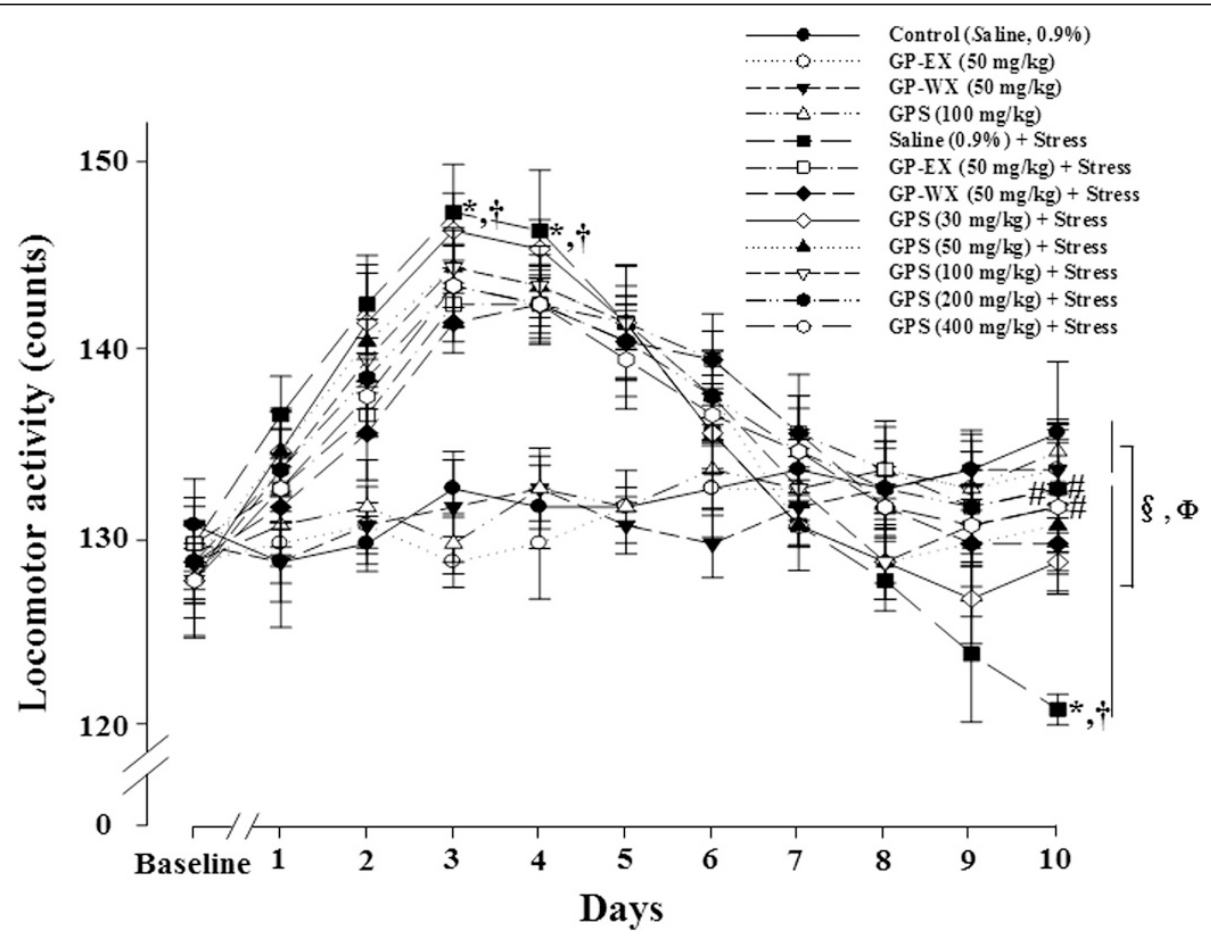

Fig. 3 Effects of GPS on spontaneous locomotor activity after exposure to chronic EF stress in mice. Mice (ICR, male, 25-30 g) were orally treated with GPS (30, 50, 100, 200 and 400 mg/kg), GP-WX (50 mg/kg), GP-EX (50 mg/kg, positive control) or saline (0.9\%) once a day for 10 days. Mice were also exposed to EF (intensity, $0.6 \mathrm{~mA}, 1 \mathrm{~s}$ every $5 \mathrm{~s}$, periods, $3 \mathrm{~min}$ ) for chronic stress for 10 days (chronic EF-stressed groups, Stress). Spontaneous locomotor activity was performed as described under the Methods section. The results are expressed as means \pm S.E.M. for 8-10 animals per groups. ${ }^{*} p<0.05$ compared with control group at each day; ${ }^{\#} p<0.05$ compared with chronic EF-stressed group at each day); ${ }^{\dagger} p<0.05$ compared with baseline (one-way ANOVA followed by Tukey's test); ${ }^{\S} p<0.05$ compared with between the un-stressed groups and stressed groups (two-way ANOVA followed by Tukey's test); ${ }^{\Phi} p<0.05$ compared with day-1 group between the three factors, doses, un-stressed and stressed stimuli, and days (three-way ANOVA followed by Tukey's test) 
and serotonin in the brain, compared with control group (GPS at $100 \mathrm{mg} / \mathrm{kg}$ was shown as a representative) (Table 1 ). Dopamine levels significantly decreased by $26.6 \%(p<0.05)$ after being exposed to EF stress for 10 days, compared with control group, and they increased by $18.0 \%(p<0.05)$ and $16.6 \%(p<0.05)$, respectively by treatment with GPS (100 and $200 \mathrm{mg} / \mathrm{kg}$ ), compared with chronic EF-stressed group (Table 1). However, GPS (30, 50 and $400 \mathrm{mg} / \mathrm{kg}$ ) did not show significant effects $(8.4 \%, 11.4 \%$ and $11.9 \%$; $p>0.05)$ (Table 1). The levels of dopamine $(\mathrm{F}=6.233$, $p<0.05)$ and serotonin $(\mathrm{F}=8.865, p<0.05)$ of the stressed groups also showed the significant differences between the un-stressed groups and stressed groups (Table 1). In addition, dopamine levels increased by $19.1 \%(p<0.05)$ by GP-WX $(50 \mathrm{mg} / \mathrm{kg})$, compared with chronic EFstressed group (Table 1).

Serotonin levels also decreased by $25.1 \%(p<0.05)$ by chronic EF stress, compared with control group (Table 1). However, serotonin levels were reduced by chronic EF stress increased by $17.6 \%(p<0.05)$ and $15.7 \%(p<0.05)$, respectively by treatment with GPS (100 and $200 \mathrm{mg} / \mathrm{kg})$, compared with chronic EF-stressed group (Table 1).
However, GPS (30, 50 and $400 \mathrm{mg} / \mathrm{kg}$ ) did not show significant effects $(6.3 \%, 9.1 \%$ and $10.5 \% ; p>0.05)$ (Table 1$)$. In addition, serotonin levels increased by $16.1 \%(p<0.05)$ by treatment with GP-WX $(50 \mathrm{mg} / \mathrm{kg})$, compared with chronic EF-stressed group (Table 1).

\section{Effects of GPS on the levels of corticosterone in the serum} In the un-stressed groups, the levels of corticosterone in the serum were not altered by treatment with GPS (30$400 \mathrm{mg} / \mathrm{kg}$ ) for 10 days, compared with control group (GPS at $100 \mathrm{mg} / \mathrm{kg}$ was shown as a representative) (Table 1 ). Corticosterone levels increased by $26.3 \%(p<0.05)$ by exposure to chronic EF stress for 10 days, compared with control group (Table 1). The levels of corticosterone also showed the significant differences between the un-stressed groups and stressed groups $(\mathrm{F}=3.454, p<0.05)$ (Table 1 ). However, corticosterone levels decreased by $16.1 \%$ $(p<0.05)$ and $15.8 \%(p<0.05)$, respectively by treatment with GPS (100 and $200 \mathrm{mg} / \mathrm{kg}$ ) for 10 days, compared with chronic EF-stressed group (Table 1). However, GPS (30, 50 and $400 \mathrm{mg} / \mathrm{kg}$ ) did not show significant effects (6.1\%, $8.4 \%$ and $14.1 \% ; p>0.05$ ) (Table 1 ).

Table 1 Effects of GPS on the levels of dopamine, serotonin and corticosterone

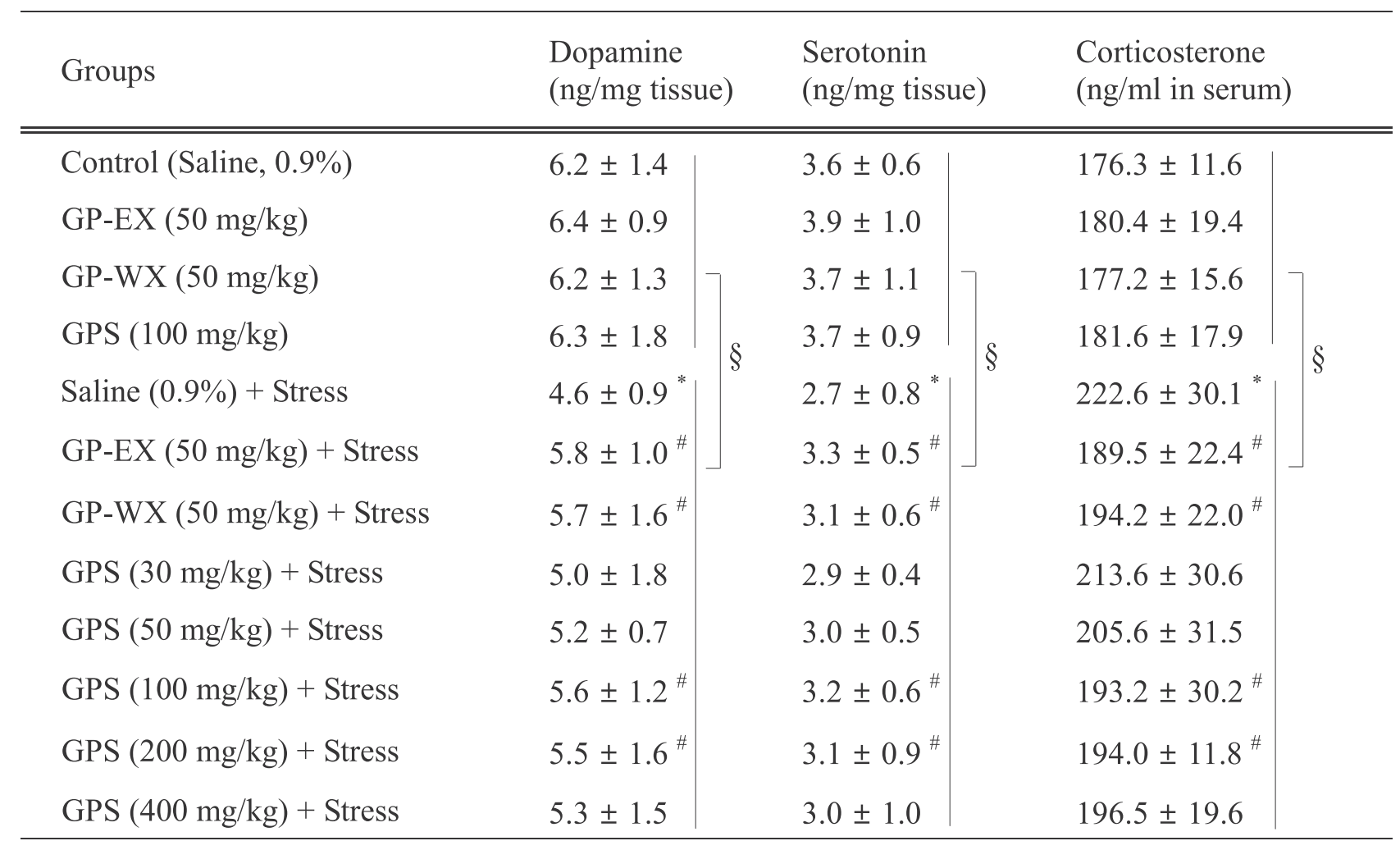

Mice (ICR, male, 20-25 g) were orally treated with GPS (30-400 mg/kg), GP-WX (50 mg/kg), GP-EX (50 mg/kg, positive control) or saline (0.9 \%) once a day for 10 days. Mice were also exposed to EF stimuli (intensity, $0.6 \mathrm{~mA}, 1 \mathrm{~s}$ every $5 \mathrm{~s}$, periods, $3 \mathrm{~min}$ ) for chronic stress for 10 days (chronic EF-stressed group, Stress). The brains were removed, and the levels of dopamine and serotonin in brain were determined by an HPLC method. The blood samples were collected on the last day of the behavioral tests, and the levels of corticosterone in serum were determined by an enzyme-linked immunosorbent assay kit. GPS (100 mg/kg) was shown as a representative of the un-stressed groups. The results are expressed as means \pm S.E.M. for $8-10$ animals per group. ${ }^{*} p<0.05$ compared with control group; ${ }^{\#} p<0.05$ compared with chronic EF-stressed group (Stress) (one-way ANOVA followed by Tukey's test). ${ }^{5} p<0.05$ compared with between the un-stressed groups and stressed groups (two-way ANOVA followed by Tukey's test) 
The levels of corticosterone were also reduced by $15.0 \%$ $(p<0.05)$ by treatment with GP-WX $(50 \mathrm{mg} / \mathrm{kg})$, compared with chronic EF-stressed group (Table 1).

\section{Effects of GPS on c-Fos-immunoreactive cells in the PVN} GPS (30-400 mg/kg) for 10 days did not alter the expression of c-Fos (GPS at $100 \mathrm{mg} / \mathrm{kg}$ was shown as a representative) (Fig. 4). The expression of c-Fos protein in the PVN markedly increased to $188 \%(p<0.01)$ by exposure to chronic EF stress for 10 days, compared with control group (Fig. 4a and b). However, c-Fos expression was reduced by treatment with GPS $(50-400 \mathrm{mg} / \mathrm{kg}$ ) for 10 days in the PVN (Fig. 4a). The number of c-Fos-immunoreactive cells in the PVN, which increased by chronic EF stress, significantly decreased by $19.4 \%(p<0.05), 23.6(\mathrm{p}<0.05)$, $30.2 \%(p<0.01), 24.3 \%(p<0.05)$ and $24.0 \%(p<0.05)$, respectively by treatment with GPS $(30,50,100,200$ and $400 \mathrm{mg} / \mathrm{kg}$ ), compared with chronic EF-stressed group (Fig. 4b). c-Fos expression of the stressed groups also significantly increased compared with the un-stressed groups $(\mathrm{F}=23.192, p<0.05)$ (Fig. 4b).

In addition, cFos expression was reduced by treatment with GP-WX (50 mg/kg) for 10 days in the PVN (Fig. 4a) and the increased number of c-Fos-immunoreactive cells by chronic EF stress was significantly reduced by $28.7 \%$ $(p<0.05)$ by treatment with GP-WX $(50 \mathrm{mg} / \mathrm{kg})$, compared with chronic EF-stressed group (Fig. 4b).

\section{Discussion}

Chronic stress-induced anxiety disorders are currently one of the most important public health concerns. Recently, GP-EX has been reported to have an ameliorating effect on chronic EF stress-induced anxiety disorders through the elevated plus-maze and marble burying tests in mice [6]. In this study, the anxiolytic effects of the gypenoside-enriched components, GPS, and GP-WX on chronic EF stress-induced anxiety disorders were investigated. In addition, their anxiolytic functions were compared
(A)

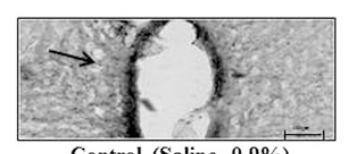

Control (Saline, $0.9 \%$ )

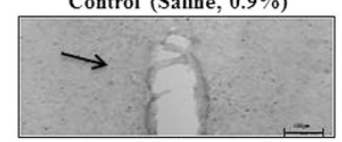

GP-WX $(50 \mathrm{mg} / \mathrm{kg})$

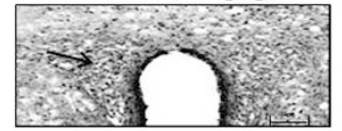

Saline $(0.9 \%)+$ Stress

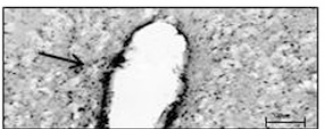

GP-WX $(50 \mathrm{mg} / \mathrm{kg})+$ Stress

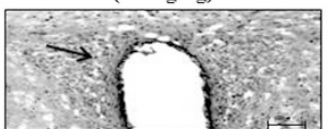

GPS $(50 \mathrm{mg} / \mathrm{kg})+$ Stress

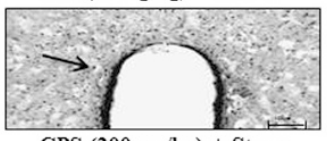

GPS $(200 \mathrm{mg} / \mathrm{kg})+$ Stress

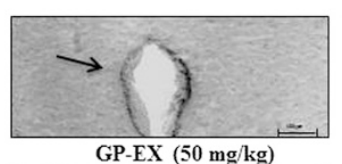

GP-EX $(50 \mathrm{mg} / \mathrm{kg})$

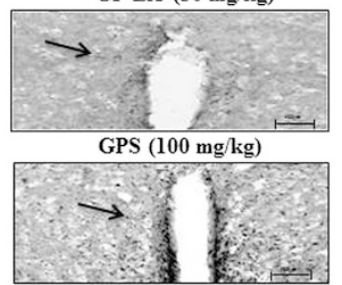

GP-EX $(50 \mathrm{mg} / \mathrm{kg})+$ Stress

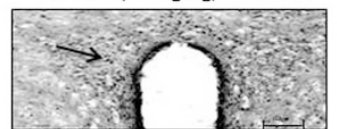

GPS $(30 \mathrm{mg} / \mathbf{k g})+$ Stress

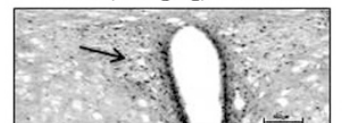

GPS $(100 \mathrm{mg} / \mathrm{kg})+$ Stress

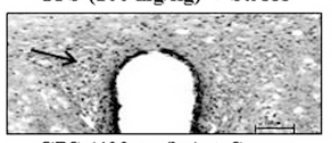

GPS $(400 \mathrm{mg} / \mathrm{kg})+$ Stress
(B)

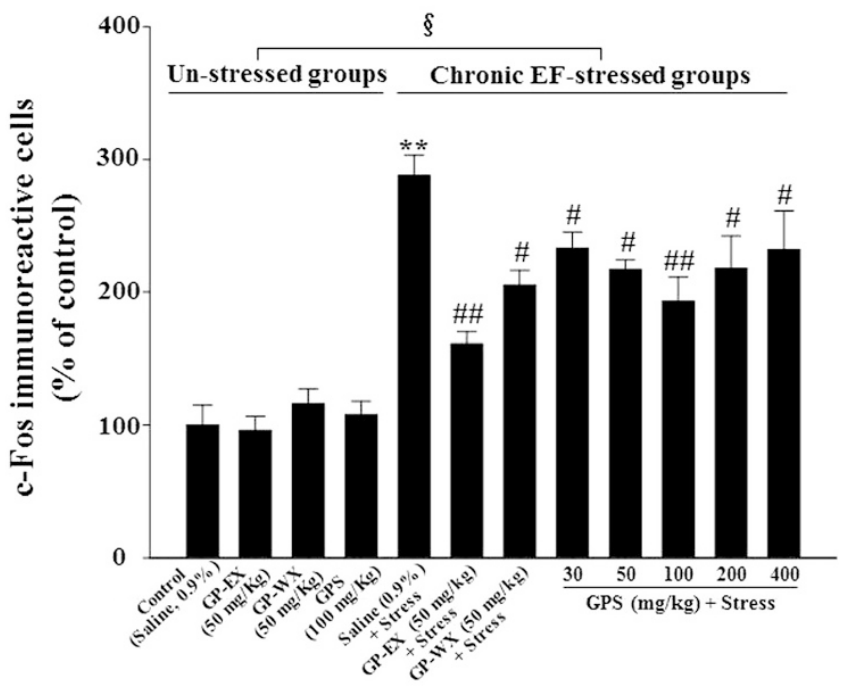

Fig. 4 Representative photographs illustrating the effects of GPS on c-Fos-immunoreactive cells in the PVN (a) and the number of c-Fos-immunoreactive cells in the PVN (b). Mice (ICR, male, 25-30 g) were orally treated with GPS (30, 50, 100, 200 and 400 mg/kg), GP-WX (50 mg/kg), GP-EX (50 mg/kg, positive control) or saline (0.9\%) once a day for 10 days. Mice were also exposed to EF stimuli (intensity, $0.6 \mathrm{~mA}, 1 \mathrm{~s}$ every $5 \mathrm{~s}$, periods, 3 min) for chronic stress for 10 days. GPS (100 mg/kg) was shown as a representative of the un-stressed groups. a Immunoblots of lysates from the brain were probed with c-Fos antibodies, and the total c-Fos-immunoreactive cells were measured as described under the Methods section. The arrow indicates nucleus of c-Fos-positive neurons. Scale bar is $100 \mu \mathrm{m}$. $\mathbf{b}$ The number of c-Fos-immunoreactive cells was counted in the PVN and was expressed as a percentage of the control groups. The number of c-Fos-immunoreactive cells of the control groups was $24 \pm 4$ cells per section. The results are expressed as means \pm S.E.M. for 8 animals per group. ${ }^{* *} p<0.01$ compared with control group; ${ }^{\#} p<0.05$, ${ }^{\# \#} p<0.01$ compared with chronic EF-stressed group (one-way ANOVA followed by Tukey's test); ${ }^{\S} p<0.05$ compared with between the un-stressed groups and stressed groups (two-way ANOVA followed by Tukey's test) 
with those by GP-EX as a positive control [6], in order to investigate whether GPS was the main functional components for anxiety disorders.

The anxiety behavior in the elevated plus-maze test is expressed as a passive avoidance in response to a potential threat. In contrast, the defensive burying behavior in the marble burying test represents an active coping strategy in response to a discrete threat [20]. In addition, spontaneous locomotor activity is reduced in response to anxiety disorders and sedation [9].

GPS $(100-400 \mathrm{mg} / \mathrm{kg})$ has beneficial effects on mouse model of PD [12, 13]. Therefore, GPS at the ranges of $30-400 \mathrm{mg} / \mathrm{kg}$ was selected to examine the ameliorating effects on chronic stress-induced anxiety disorders in mice. In our study, treatment with GPS at $100-200 \mathrm{mg} / \mathrm{kg}$ significantly recovered chronic EF stress-reduced the number of open arm entries and the time spent on open arms (Fig. 1a and b). Closed arm entries, which are a measure of motor activity in elevated plus-maze, were slightly decreased by GPS, but it was not significant (data not shown). The ratios of the number of open arm entries to the total arm entries, which is considered to be related to the level of anxiety, were significantly reduced in the chronic EF-stressed groups. GPS (100-400 mg/kg) reduced the number of marbles buried which was increased by chronic EF stress (Fig. 2). In addition, the spontaneouse locomotor activity is related to dopamine levels [21]. At early periods of 2-3 days, the locomotor activities of control group were increased by acute stress, which was caused by increasing dopamine levels [22]. However, at later periods of 6-10 days, anxiety and/or depression were induced by the repeated chronic stress, and in these states, the spontaneous locomotor activities were gradually decreased below than control group [6]. In this study, GPS (100-200 mg/kg) reversed spontaneous locomotor activity for day- 6 to day-10. GPS also showed the significant protective effects between the un-stressed groups and stressed groups at day-10, compared with day-1. These results indicate that GPS shows the anxiolytic functions without decreasing spontaneous locomotor activity in mice. In addition, treatment with GP-WX $(50 \mathrm{mg} / \mathrm{kg})$ exhibited an ameliorating effect on chronic EF stressinduced anxiety disorders which was respectively measured by elevated plus-maze, marble burying and locomotor activity (Figs. 1, 2 and 3).

Chronic stress-induced anxiety disorders decreases dopamine and serotonin levels in the brain [2]. Chronic stressinduced anxiety-like states maintains the increased levels of corticosterone compared with the un-stressed states. However, the levels of corticosterone induced by chronic stress are lower than those by acute stressed states and these reduced levels of corticosterone lead to regulate dopamine and serotonin levels [2]. In addition, anxiolytic-like behavioral responses in the elevated plus-maze test are mediated by the serotonin receptor functions [5]. The number of marbles buried is reduced by benzodiazepine receptor agonist (diazepam) and serotonin reuptake inhibitor (fluvoxamine) [6]. Dopamine receptor blocking agents inhibit spontaneous locomotor activity [21]. In this study, dopamine and serotonin levels were reduced by chronic EF stress in the brain, and they were increased by treatment with GPS (100-200 mg/kg) (Table 1). In contrast, the serum levels of corticosterone obviously increased by chronic EF stress, and they were also reversed by treatment with GPS (100-200 mg/kg) (Table 1). These results suggest that GPS can effectively ameliorate chronic stress-induced anxiety disorders by modulating the levels of dopamine, serotonin and corticosterone. In addition, the same patterns were also obtained by treatment with GP-WX $(50 \mathrm{mg} / \mathrm{kg})$ (Table 1). The serum levels of corticosterone induced by chronic EF stress were also modulated by treatment with GP-WX (50 mg/kg) (Table 1).

Among the Fos family members, FosB levels increase after being exposed to chronic stress [23], and the c-Fos expression in the PVN regions increases by chronic and acute stress in mice and rats [3, 4]. In addition, the PVN is essential for regulation of the HPA axis and may influence HPA axis hormone secretion such as corticosterone $[24,25]$. GPS $(30-400 \mathrm{mg} / \mathrm{kg})$ significantly reduced c-Fos expression induced by chronic EF stress (Fig. 4), and also reduced the serum levels of corticosterone (Table 1). These results suggest that the modulation of c-Fos expression by GPS plays a role in the protective function in anxiety disorders induced by chronic EF stress. Chronic EF stress-induced c-Fos expression also decreased by GP-WX (50 $\mathrm{mg} / \mathrm{kg}$ ) in the PVN regions (Fig. 4).

In this study, GPS (30 and $50 \mathrm{mg} / \mathrm{kg}$ ) exhibited the trend of anxiolytic effects on chronic EF stress-induced anxiety disorders, but it was not significant. GPS $(400 \mathrm{mg} / \mathrm{kg}$ ) also showed the anxiolytic effects on chronic EF stressinduced anxiety disorders. However, GPS at the ranges of $100-200 \mathrm{mg} / \mathrm{kg}$ showed a maximal efficacy on anxiolytic functions, as compared with GPS (30, 50 and $400 \mathrm{mg} / \mathrm{kg}$ ). The high dose of GPS $(400 \mathrm{mg} / \mathrm{kg})$ might not be adopted for an oral administration in mice and the similar results have been also observed in a previous study [13].

GPS has been found to protect aortic endothelial cells against oxidative damage [26]. GPS shows the protective effects on oxidative stress induced by glutamate-induced neurotoxicity [27]. In addition, oxidative stress may cause the anxiety-related behaviors and the antioxidants could be used for the prevention or reduction of the high anxiety [28]. It is therefore possible to explain that the symptoms of anxiety disorders induced by chronic EF stress can be relieved by treatment with GPS and GP-WX, which has been closely mediated by the anti-oxidative activity in rodents.

In the present study, GPS at $100-200 \mathrm{mg} / \mathrm{kg}$, but not $50 \mathrm{mg} / \mathrm{kg}$, showed an equivalent efficacy on the 
anxiety behavioral tests, as compared with GP-EX at $50 \mathrm{mg} / \mathrm{kg}$. However, GP-WX (50 mg/kg) showed a slightly less effect on the chronic stress-induced anxiety disorders than GP-EX (50 mg/kg) and GPS (100-200 mg/kg). GPWX or GP-EX has been identified to have many kinds of GPS, flavonoids, polysaccharides, vitamins and amino acids [10]. GPS, GP-WX and GP-EX showed the different chromatogram patterns by the levels of bioactive components (the supplement data): GP-EX contained mainly the gypenoside, flavonoid and polysaccharide components, and GP-WX also contained the polysaccharide components. The levels of gypenoside components in GPS were higher than those in GP-EX. In addition, the levels of polysaccharide components in GP-EX were higher than those in GP-WX, which was much higher than those in GPS. The polysaccharides of GP-EX show various functions, including anti-aging, anti-fatigue, improvingimmune competence and potential anti-oxidant $[29,30]$. Polysaccharides supplementation also has beneficial effects on attenuating the oxidative stress induced by exhaustive exercise in rats [31]. These results suggest that GPS plays an important role in anti-anxiety disorders, and that besides GPS, the bioactive components including flavonoids and polysaccharides in GP-EX or GP-WX also show the anxiolytic-like functions. However, the intensities of anxiolytic-like functions between GPS and polysaccharides from GP need to be studied.

GPS $(50-400 \mathrm{mg} / \mathrm{kg})$ for 10 days does not exhibit the adverse effects, such as weight loss, diarrhea, vomiting and death. The values of $\mathrm{LD}_{50}$ of total GPS are $755-838 \mathrm{mg} / \mathrm{kg}$ (injected into the abdominal cavity) and $402 \mathrm{mg} / \mathrm{kg}$ $( \pm 18.2 \mathrm{mg} / \mathrm{kg}$, i.p. $)$ in mice [32]. GP-WX $(750 \mathrm{mg} / \mathrm{kg})$ does not also produce any significant toxicity in rats during 6-month period of treatment [33]. The lethal dose of total GP-EX is $1800-2000 \mathrm{mg} / \mathrm{kg}$ (p.o.) (data not shown). These results suggest that GPS might be safe more than GP-EX although GPS shows a less efficacy on chronic stress-induced anxiety disorders than GP-EX. However, GPS, GP-WX and GP-EX have been proved as safe herbal materials for treatment with animal models $[6,10]$.

\section{Conclusion}

GPS (100-200 mg/kg) exhibited the anxiolytic effects on chronic EF stress-induced anxiety behaviors in mice, which was evaluated by the elevated plus-maze and marble burying and spontaneous locomotor activity tests by modulating the levels of dopamine, serotonin, corticosterone and c-Fos expression. GP-WX also showed the anxiolytic activity on chronic EF stress-induced anxiety behaviors in mice, but it was lower activities than those obtained by GPS. GPS may serve as a phytonutrient in chronic stress-induced anxiety disorders and clinical tests of GPS need to be conducted further.
Availability of data and materials

Not applicable.

\section{Abbreviations}

GPS: Gypenosides; EF stress: Electric footshock stress; PVN: Paraventricular nuclei; GP: Gynostemma pentaphyllum; GP-EX: Ethanol extract from GP; GP-WX: Water extract from GP; PD: Parkinson's disease; HIAA: 5-

Hydroxyindoleacetic acid.

\section{Competing interests}

The authors declare that there is no conflict of interests regarding the publication of this paper.

\section{Authors' contributions}

TTZ, KSS and HSC performed drug treatments, behavioral tests, immunohistochemical analyses and statistical analyses, and wrote a part of the manuscript. TTZ, KSS and MKL carried out the study and analyzed the data. MKL conceived, designed and supervised all the experiments, and prepared the manuscript. All authors read and approved the final manuscript.

\section{Acknowledgements}

This research was financially supported by the Ministry of Knowledge Economy (MKE) and Korea Institute for Advancement of Technology (KIAT) through the Inter-ER Cooperation Projects (R0002021) (2014) and by the Research Center for Bioresource and Health, MKE and KIAT (2014), Republic of Korea.

The authors wish to thank the Ministry of Knowledge Economy (MKE) and Korea Institute for Advancement of Technology (KIAT) through the Inter-ER Cooperation Projects (R0002021) (2014), and the Research Center for Bioresource and Health, MKE and KIAT (2014), Republic of Korea.

\section{Author details}

${ }^{1}$ College of Pharmacy and Research Center for Bioresource and Health, Chungbuk National University, Cheongju, Chungbuk 28644, Republic of Korea. ${ }^{2}$ Department of Food and Nutrition, Chungcheong University, Cheongju, Chungbuk 28171, Republic of Korea.

Received: 8 January 2015 Accepted: 9 September 2015

Published online: 14 September 2015

\section{References}

1. Finlay JM, Zigmond MJ, Abercrombie ED. Increased dopamine and norepinephrine release in medial prefrontal cortex induced by acute and chronic stress: Effects of diazepam. Neuroscience. 1995;64:619-28.

2. Sheikh N, Ahm A, Siripurapu KB, Kuchibhotla VK, Singh S, Pali G. Effect of Bacopa monniera on stress induced changes in plasma corticosterone and brain monoamines in rats. J Ethnopharmacol. 2007;111:671-6.

3. Chen $X$, Herbert J. Regional changes in c-fos expression in the basal forebrain and brainstem during adaptation to repeated stress: correlations with cardiovascular, hypothermic and endocrine. Neuroscience. 1995;64:675-85.

4. Imaki T, Katsumata H, Konishi SI, Kasagi Y, Minami S. Corticotropin-releasing factor type-1 receptor mRNA is not induced in mouse hypothalamus by either stress or osmotic stimulation. J Neuroendocrinol. 2003;15:916-24.

5. Verma P, Hellemans KGC, Choi FY, Yu W, Weinberg J. Circadian phase and sex effects on depressive/anxiety-like behaviors and HPA axis responses to acute stress. Physiol Behav. 2010;99:276-85.

6. Choi HS, Zhao TT, Shin KS, Kim SH, Hwang BY, Lee CK. Anxiolytic effects of herbal ethanol extract from Gynostemma pentaphyllum after exposure to chronic stress in mice. Molecules. 2013;18:4342-56.

7. Lin D, Parsons LH. Anxiogenic-like effect of serotonin $1 B$ receptor stimulation in the rat elevated plus-maze. Pharmacol Biochem Behav. 2002;71:581-7.

8. Sugimoto $Y$, Tagawa N, Kobayashi $Y$, Hotta $Y$, Yamada J. Effects of the serotonin and noradrenaline reuptake inhibitor (SNRI) milnacipran on marble burying behavior in mice. Biol Pharm Bull. 2007;30:2399-401.

9. Han HS, Ma Y, Eun JS, Li RH, Hong JT, Lee MK, et al. Anxiolytic-like effects of sanjoinine A isolated from Zizyphi spinosi semen: possible involvement of GABAergic transmission. Pharmacol Biochem Behav. 2009;92:206-13.

10. Razmovski-Naumovski V, Huang THW, Tran VH, Li GQ, Duke CC. Chemistry and pharmacology of Gynostemma pentaphyllum. Phytochem Rev. 2005;14:197-219. 
11. Choi HS, Park MS, Kim SH, Hwang BY, Lee CK, Lee MK. Neuroprotective effects of herbal ethanol extracts from Gynostemma pentaphyllum in the 6-hydroxydopamine-lesioned rat model of Parkinson's disease. Molecules. 2010;15:2814-24.

12. Shin KS, Zhao TT, Choi HS, Hwang BY, Lee CK, Lee MK. Effects of gypenosides on anxiety disorders in MPTP-lesioned mouse models of Parkinson's disease. Brain Res. 2014;1567:57-65.

13. Wang P, Niu L, Gao L, Li WX, Jia D, Wang XL, et al. Neuroprotective effect of gypenosides against oxidative injury in the substantia nigra of a mouse model of Parkinson's disease. J Int Med Res. 2010;38:1084-92.

14. Lv Y, Yang XB, Zhao Y, Ruan Y, Yang Y, Wang ZZ. Separation and quantification of component monosaccharides of the tea polysaccharides from Gynostemma pentaphyllum by HPLC with indirect UV detection. Food Chem. 2009;112:742-6.

15. Matuszewich L, Mcfadden LM, Friedman RD, Frye CA. Neurochemical and behavioral effects of chronic unpredictable stress. Behav Pharmacol. 2014;25:557-66.

16. Husain GM, Chatterjee SS, Singh PN, Kumar V. Antiaggressive activity of standardized extract of Indian Hypericum perforatum L. Pharmacologyonlne. 2009;1:432-44.

17. Garcia AMB, Cardenas FP, Morato S. Effect of different illumination levels on rat behavior in the elevated plus-maze. Physiol Behav. 2005;85:265-70.

18. Chotiwat C, Harris RBS. Increased anxiety-like behavior during the post-stress period in mice exposed to repeated restraint stress. Horm Behav. 2006;50:489-95.

19. Satoh K, Nonaka R, Ohashi N, Shimizu M, Oshio S, Takeda K. The effects of in utero exposure to a migrant, 4,4'-butylidenebis (6-t-butyl-m-cresol), from nitrile-butadiene rubber gloves on monoamine neurotransmitter in rat. Biol Pharm Bull. 2008:31:2211-5.

20. Gorton LM, Vuckovic MG, Vertelkina N, Petzinger GM, Jakowec MW, Wood RI Exercise effects on motor and affective behavior and catecholamine neurochemistry in the MPTP-lesioned mouse. Behav Brain Res. 2010;213:253-62.

21. Vallone D, Picetti R, Borrelli E. Structure and function of dopamine receptors, Neurosci Biobehav Rev. 2000;24:125-32.

22. Zhao TT, Shin KS, Choi HS, Lee MK. Effects of Gypenosides on Acute Stress in Mice. Nat Prod Sci. 2013;19:337-41.

23. Stamp J, Herbert J. Corticosterone modulates autonomic responses and adaptation of central immediate-early gene expression to repeated restraint stress. Neuroscience. 2001;107:465-79.

24. Smith SM, Vale WW. The role of the hypothalamic-pituitary-adrenal axis in neuroendocrine responses to stress. Dialogues Clin Neurosci. 2006:8:383-95.

25. Windle RJ, Kershwa YM, Shanks N, Wood SA, Lightman SL, Ingram CD. Oxytocin attenuates stress-induced c-fos mRNA expression in specific forebrain regions associated with modulation of hypothalamo-pituitaryadrenal activity. J Neurosci. 2004;24:2974-82

26. Tanner MA, Bu X, Steimle JA, Myers PR. The direct release of nitric oxide by gypenosides derived from the herb Gynostemma pentaphyllum. Nitric Oxide. 1999:3:359-65.

27. Shang LS, Liu JC, Zhu QJ, Zhao L, Feng YX, Wang WP, et al. Gypenosides protect primary cultures of rat cortical cells against oxidative neurotoxicity. Brain Res. 2006;1120:63-174.

28. Rammal H, Bouayed J, Younos C, Soulimani R. Evidence that oxidative stress is linked to anxiety-related behaviour in mice. Brain Behav Immun. 2008:22:1156-9.

29. Wang ZI, Luo DH. Antioxidant activities of different fractions of polysaccharide purified from Gynostemma pentaphyllum Makino. Carbohydr Polym. 2007:68:54-8.

30. Yang XB, Zhao Y, Yang Y, Ruan Y. Isolation and characterization of immunostimulatory polysaccharide from an herb tea, Gynostemma pentaphyllum Makino. J Agric Food Chem. 2008;56:6905-9.

31. Wang HF, Li CJ, Wu XL, Lou XJ. Effects of Gynostemma pentaphy/lum (Thunb.) Makino polysaccharides supplementation on exercise tolerance and oxidative stress induced by exhaustive exercise in rats. Afr J Agric Res. 2012;7:2632-8.

32. Guo WY, Wang WX. In: Cultivation and Utilisation of Gynostemma pentaphyllum. Beijing: Publishing House of Electronics, Science and Technology University; 1993:1-261.

33. Attawish A, Chivapat S, Phadungpat S, Bansiddhi J, Techadamrongsin Y, Mitrijit O. Chronic toxicity of Gynostemma pentaphyllum. Fitoterapia. 2004;75:539-51.

\section{Submit your next manuscript to BioMed Central and take full advantage of:}

- Convenient online submission

- Thorough peer review

- No space constraints or color figure charges

- Immediate publication on acceptance

- Inclusion in PubMed, CAS, Scopus and Google Scholar

- Research which is freely available for redistribution

Submit your manuscript at www.biomedcentral.com/submit 